\title{
Representations of the French language / culture among Qatari students enrolled in the minor of French at Qatar University
}

\section{[Les représentations de la langue/culture française chez les étudiantes qatariennes inscrites en mineure de français à l'Université du Qatar]}

\author{
Elie Alrabadi
}

DOI: $10.18355 / X L .2021 .14 .04 .10$

\begin{abstract}
This research is interested in the representations that Qatari students, enrolled in the French minor at Qatar University, have toward the French language/culture. The objective of this research is to analyze these representations as well as their influences on the motivations and attitudes of these students towards learning French. To achieve this goal, we conducted a survey among these students. The results this survey show that French generally receives positive representations, which should develop attitudes favorable to its learning.
\end{abstract}

Key words: Qatar, sociolinguistic situation, language practices, French language, representations, motivations, attitudes

\section{Résumé}

Cette recherche s'intéresse aux représentations que se font les étudiantes qatariennes, inscrites en mineure de français à l'université du Qatar, de la langue/culture française. Son objectif est d'analyser ces représentations ainsi que leurs influences sur les motivations et les attitudes de ces étudiantes envers l'apprentissage du français. Pour ce faire, nous avons mené une enquête auprès de ces étudiantes dont les résultats montrent que le français reçoit généralement des représentations positives ce qui devrait développer des attitudes favorables à son apprentissage.

Mots-clés : Qatar, situation sociolinguistique, pratiques langagières, langue française, représentations, motivations, attitudes

\section{Introduction}

Les approches communicatives et la perspective actionnelle placent l'apprenant au centre du processus d'enseignement/apprentissage des langues étrangères (LE). Il devient ainsi l'acteur le plus important en classe de langue dans la mesure où tout part et revient à lui (Richterich, 1985: 104). L'identification et l'analyse de ses besoins, de ses objectifs, de ses motivations, de ses attitudes ainsi que de ses représentations envers la langue cible deviennent impératives dans la mise en place d'une pédagogie réussie. L'apprentissage d'une LE ne se limite dorénavant plus aux seuls facteurs cognitifs. La dimension affective, qui englobe les représentations, les motivations et les attitudes des apprenants, joue également un rôle aussi important dans ce processus (Arnold 2006: 407). Le Cadre Européen commun de référence pour les langues (2002: 84) confirme également l'importance de la prise en compte des aspects affectifs, et précise que «l'activité de communication des utilisateurs/apprenants est non seulement affectée par leurs connaissances, leur compréhension et leurs aptitudes mais aussi par des facteurs personnels liés à leur personnalité propre et caractérisés par les attitudes, les motivations, les valeurs, les croyances et les types de personnalité qui constituent leur identité ».

Chaque langue/culture véhicule un certain nombre de représentations qui sont présentes dans la perception des apprenants avant même de commencer son apprentissage. Pour Calvet (1999: 165), une langue est avant tout « un ensemble de

XLinguae, Volume 14 Issue 4, October 2021, ISSN 1337-8384, eISSN 2453-711X 
pratiques et de représentations ». Ces représentations que les apprenants se font d'une LE, de ses locuteurs, de sa culture, de ses normes, de ses caractéristiques, des pays où la langue est parlée et de son statut au regard des autres langues, influencent largement leurs choix d'apprendre telle ou telle langue ainsi que leurs attitudes vis-àvis de la langue en question (Zarate, 1993 ; Dabène, 1994, 1997 ; Moore, 2001 ; Castellotti et Moore, 2002 ; Alrabadi, 2012, 2017). A ce propos, Moore (2001: 11), souligne que toutes les recherches en didactique des LE lient «les attitudes et les représentations au désir d'apprendre les langues, et à la réussite ou à l'échec de l'apprentissage ». Les représentations positives envers la langue/culture cible conduisent tout naturellement à «des attitudes xénophiles qui s'expriment généralement par des comportements et des pratiques d'ouverture à l'Autre, alors que les représentations négatives mènent à des comportements qui s'extériorisent à travers des comportements xénophobes de rejet et de refus à l'Autre »(GohardRadenkovic et al., 2003 29-30).

Les représentations positives renforcent par conséquent la motivation des apprenants et développent une attitude favorisant l'apprentissage et l'emploi de la LE. Dans le cas contraire, elles constituent une source de démotivation et de freins qui entravent l'apprentissage de la LE (Py, 2004: 15 ; Alrabadi, 2012: 51).

Dans ce travail de recherche, nous nous intéressons tout particulièrement aux représentations que les étudiantes qatariennes inscrites au programme de mineure de français à l'université du Qatar, se font de la langue/culture française, de la France et des Français. Nous essayerons de répondre aux questions suivantes: Quel est le statut du français au Qatar? Quelle place occupe-t-il dans le pays ? Quels seraient les objectifs et les motivations de ces étudiantes ? Quelles seraient les représentations que ces étudiantes se font de la langue/culture française, des Français et de la France ? Quel serait l'impact de ces représentations sur leurs attitudes envers l'apprentissage du français?

Pour ce faire, nous aborderons le concept de représentations en didactique des LE. Nous exposerons la situation sociolinguistique et démographique dans le pays. Nous présenterons notre méthodologie ainsi que notre contexte de recherche. Nous analyserons enfin les résultats d'une enquête réalisée auprès des étudiantes inscrites au programme de mineure de français à l'Université du Qatar.

La présente recherche requiert une importance particulière de par le fait qu'elle constitue la première recherche scientifique s'intéressant aux représentations du français au Qatar.

\section{Représentations en didactique des LE :}

Nombreuses sont les recherches qui ont souligné l'importance des représentations et leur influence sur la détermination des motivations et des attitudes des apprenants vis-à-vis de la langue/culture cible (Abdallah-Pretceille, 1986; Zarate, 1986, 1993, 1997 ; Candelier et Brennecke, 1993 ; Porcher, 1995, Cain et De Pietro, 1997 ; Candelier, 1997 ; Dabene, 1997; Matthey, 1997, 2000, Puren, 1997 ; Muller, 1998; Muller et De Pietro, 2001; Paganini, 1998 ; Beacco, 2001 ; Berger, 2001 ; Billiez et Millet, 2001 ; Moore, 2001 ; Castellotti et Moore, 2002 ; Cavalli et Coletta, 2002 ; Auger, 2003 ; Gohard-Radenkovi, et al., 2003 ; Py, 2004 ; Amossy et Herschberg-Pierrot, 2007 ; Alrabadi, 2012, 2017).

En effet, la notion de représentation(s) est largement présente dans toutes les sciences humaines, y compris dans la didactique des LE où elle acquiert une importance particulière étant donné que la classe de LE est un des lieux privilégiés d'interculturel, c'est-à-dire de rencontre entre la langue/culture maternelle des apprenants, et celle de la LC.

Terme emprunté à la psychologie sociale, une représentation est « une forme de connaissance socialement élaborée et partagée, ayant une visée pratique et concourant à la construction d'une réalité commune à un ensemble social » (Jodelet, 
1989: 63). Elle montre l'adhésion d'un individu à un groupe d'appartenance et régit notre relation au monde et aux autres, modelant ainsi les conduites et les interactions sociales (Moscovici, 1989: 3 ; Amossy et Herschberg-Pierrot, 2010: 50).

En didactique des LE « la notion de la représentation devient opératoire, car elle permet de rendre compte des sources multiples mobilisées dans un processus d'apprentissage et d'enseignement des langues » (Castellotti et Moore, 2002: 11).

Dabène (1994: 50-52) souligne qu'il existe cinq critères qui déterminent le choix de l'apprentissage des LE. Les représentations se fondent souvent sur ces critères :

- L'utilité réelle ou supposée de la LC dans la vie quotidienne, scolaire, professionnelle, techniques, etc.

- Le degré de facilité/difficulté d'apprentissage de la LC en fonction de sa prononciation, de sa grammaire, de son écriture, etc.

- $\quad$ Le prestige dont jouit la LC, les pays où elle est parlée, ses locuteurs et par conséquent ceux qui l'apprennent. Ce prestige dépend du poids culturel, historique, géopolitique et économique de la LC.

- La beauté de la LC en fonction de sa prononciation et des productions artistiques qui lui sont associées.

- La sympathie/l'antipathie pour les autochtones en fonction des relations historiques (notamment coloniales) et des conflits de la politique internationale.

Il est à noter que ces représentations sont évolutives et peuvent changer d'une époque à une autre dans la mesure où elles sont engendrées par des situations politiques, économiques, diplomatiques ou sociologiques changeantes.

\section{La situation sociolinguistique du Qatar}

Avant de s'intéresser au paysage sociolinguistique du Qatar, il nous semble impératif de se pencher sur l'évolution de la situation démographique du pays.

\section{1. Démographie :}

Le Qatar, petit État très riche, situé à l'est du golfe arabo-persique, est d'une superficie de $11521 \mathrm{~km} 2$. Doha, capitale du pays, est sa principale ville, dans laquelle vit plus de la moitié de la population. Le pays a connu dans les deux dernières décennies une croissance économique et démographique exceptionnelle grâce à l'exploitation de ces énormes réserves pétro-gazières. Depuis les années 2000 et avec sa grande croissance économique, le pays investit massivement dans différents secteurs à l'échelle internationale tels que le sport et les médias (Srour-Gondon, 2013: 46). Au niveau national, il a également entamé une politique de modernisation de ses infrastructures dans tous les domaines (construction, transports, éducation, santé, culture, tourisme, hôtellerie, sport, loisirs, etc.), notamment avec l'accueil de la Coupe du monde de Football en 2022.

Selon les statistiques officielles du Qatar, la population a presque doublé deux fois en quinze ans, passant de 890000 habitants en 2005 à 2684329 habitants en $2020^{1}$. Il est à noter que l'Autorité de la Planification et des Statistiques de l'État ne publie pas de données concernant les nationalités ou les groupes ethniques des résidents étrangers. Cependant, selon un rapport d'une société de conseil privée datant de 2019², cité par Ahmad et Hilman (2020: 6), il existe quatre-vingt-quatorze nationalités différentes résidant actuellement au Qatar. Les Qatariens nationaux ne

\footnotetext{
${ }^{1}$ https://www.psa.gov.qa/en/statistics1/pages/default.aspx

${ }^{2}$ https://priyadsouza.com/population-of-qatar-by-nationality-in-2017/
}

XLinguae, Volume 14 Issue 4, October 2021, ISSN 1337-8384, eISSN 2453-711X 
représentent qu'une petite minorité d'environ $11 \%$ de l'ensemble de la population ${ }^{3}$. Généralement qualifiés, ils travaillent surtout dans les administrations et les entreprises publiques, au sein desquelles ils occupent souvent des postes de cadres dirigeants et de managers ${ }^{4}$.

Pour répondre aux besoins de sa croissance économique, le pays a dû chercher une main-d'œuvre qualifiée et non qualifiée, provenant de plusieurs pays. Selon le même rapport, ces travailleurs viennent principalement :

- $\quad$ Des pays du sud et du sud-est asiatique tels que l'Inde, le Bangladesh, le Népal, le Pakistan, les Philippines et le Sri Lanka, représentant ainsi $65 \%$ de l'ensemble de la population. A titre d'exemple, le Qatar accueille 700000 Indiens, soit $21.8 \%$ de l'ensemble de la population, 400000 Bengalis et 400000 Népalais $(12.5 \%$ de la population pour chaque communauté). Le nombre de ressortissants de chacune de ces trois communautés dépasse donc celui des Qatariens. La main-d'œuvre non qualifiée à bon marché venant de ces pays asiatiques travaille souvent sur des chantiers de construction, dans des domaines de travaux manuels, dans les hôtels, le jardinage, les restaurants, les centres commerciaux, les travaux domestiques chez des particuliers (s'occuper des enfants et du ménage, faire les chauffeurs, etc.). Ils sont recrutés avec des contrats à durée déterminée dans leur pays d'origine, par les agences de recrutement. Le recrutement des travailleurs étrangers se fait souvent selon des quotas précisés par le gouvernement qatarien et des conventions avec leurs pays d'origine.

- $\quad$ Des pays arabes tels que l'Egypte, le Soudan, la Syrie, la Jordanie, le Liban ainsi que les pays du Maghreb (la Tunisie, le Maroc et l'Algérie) avec un pourcentage de $17 \%$ de la population du Qatar.

- Des pays occidentaux qui sont également de plus en plus présents dans le pays, avec un pourcentage aux environs de $6 \%$.

Les ressortissants de ces deux catégories de la population, souvent des travailleurs qualifiés, occupent donc des postes plus rémunérés.

- Des pays africains et d'autres pays dans le monde (1 \%). Les travailleurs africains occupent souvent des fonctions semblables aux travailleurs asiatiques.

Les travailleurs étrangers n'ont pas le droit de faire venir leurs familles, sauf les travailleurs qualifiés ayant des ressources financières convenables. Il est à noter que le Qatar n'accorde sa nationalité que très rarement et dans des cas très rares, voire exceptionnels ${ }^{5}$.

Comme nous pouvons le constater, cette grande diversité démographique engendre des répercussions naturellement importantes sur la situation sociolinguistique dans le pays, sur les rapports entre les différentes langues, sur leurs représentations ainsi que sur les pratiques langagières des individus.

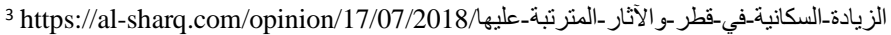

${ }^{4}$ https://www.tresor.economie.gouv.fr/Articles/a7d5a8e0-fc0b-4ae2-acda-

3efa5c8e9fce/files/be882ed0-a42a-49fe-b269-569b95dd629f

${ }^{5}$ Majed Al-Ansari, professeur de sociologie politique à l'université du Qatar, cité par le monde diplomatique (2018) : https://www.monde-diplomatique.fr/2018/10/MOUNIER_KUHN/59150
} 


\section{2. Langues en présence}

Le paysage linguistique au Qatar se distingue par la présence de plusieurs langues parlées par les différentes communautés qui y sont présentes, faisant de ce petit pays un site d'hyper-diversité linguistique "linguistic superdiversity » (Nebel, 2017: 29 ; Hilman et Ocampo Eibenschutz, 2018: 7). Cette grande diversité linguistique exige le recours à une ou plusieurs langues médiatrices assurant la communication entre les différentes communautés. Les pratiques de ces langues par les différentes communautés sont déterminées par les représentations attachées à chacune d'entre elles.

Dans le contexte qatarien, nous avons des langues considérées comme langues supercentrales (Calvet 1999, 2007: 47) telles que l'arabe, l'anglais et le français, et d'autres langues, périphériques et moins prestigieuses telles que les différentes langues parlées par les ouvriers asiatiques à titre d'exemple.

\section{2. 1. L'arabe}

Le Qatar est un pays arabophone. L'arabe s'y trouve en situation diglossique voir hétéro-glossique. Au Qatar, comme c'est le cas dans les autres pays arabes, nous pouvons distinguer trois niveaux d'arabe :

- L'arabe classique, langue officielle du pays, utilisé dans l'enseignement religieux, «très inspiré par le texte coranique, est celui des lettrés, c'est un langage châtié, élaboré, d'usage liturgique ou littéraire » (Ait-Chaalal, 2007: 56).

- L'arabe standard moderne, utilisé notamment à l'écrit, comme langue d'enseignement dans les écoles publiques, dans les médias et dans l'Administration.

- L'arabe dialectal qatarien, proche des autres dialectes des pays du Golfe, langue maternelle de la grande majorité des Qatariens, utilisé surtout à l'oral dans la communication quotidienne ${ }^{6}$. Il se côtoie et se mélange avec les autres dialectes arabes, parlés par les différentes communautés arabophones présentes dans le pays.

L'arabe, avec ses différentes formes, est utilisé par les différentes communautés arabophones et non-arabophones. Concernant les non-arabophones, il s'agit souvent d'ouvriers asiatiques venant des pays du sud et du sud-est de l'Asie. Ils sont pour la grande majorité d'entre eux semi-alphabètes et ne parlent pas l'anglais comme lingua franca (Ahmad et Hillman, 2020: 5). Dans leur communication avec les arabophones, ils utilisent une forme très simplifiée de l'arabe appelée « l'arabe pidgin du Golfe » (Avram, 2014: 8).

Dans les faits, l'arabe fait partie de l'identité culturelle des Arabes en général et de l'identité religieuse des Musulmans en particulier. Pour valoriser et renforcer sa place face à l'expansion de l'emploi de l'anglais, l'État a pris un certain nombre de mesures comme la « loi sur la protection de l'arabe de $2019^{7}$ », rendant son usage obligatoire dans les différents domaines de la vie publique.

\section{2. 2. L'anglais}

\footnotetext{
${ }^{6} \mathrm{Il}$ est à noter que les dialectes arabes sont de plus en plus utilisés à l'écrit par les jeunes dans leurs échanges sur les différents réseaux sociaux (Facebook, Twitter, Instagram, WhatsApp, etc.).

${ }^{7}$ https://diwan.gov.qa/briefing-room/news/general//g/2019/140119/lg02?sc_lang=en
}

XLinguae, Volume 14 Issue 4, October 2021, ISSN 1337-8384, eISSN 2453-711X 
L'anglais n'a pas de statut officiel au Qatar mais il est considéré de facto comme la première langue de communication entre les différentes communautés vivant dans ce pays.

Concernant son rôle dans l'enseignement, l'anglais bénéficie d'un statut très favorable. Il est enseigné comme cours obligatoire dans les écoles publiques et privées $\mathrm{du}$ pays à partir de la première année de scolarisation. Il est aussi la langue d'enseignement dans les écoles privées qui adoptent un curriculum britannique et américain, à titre d'exemple.

Concernant l'enseignement supérieur, l'anglais reste utilisé comme langue d'enseignement pour les spécialités scientifiques à l'Université du Qatar, seule université publique du pays.

Il est à noter aussi qu'il existe un certain nombre d'universités privées fondées en coopération avec d'autres pays comme l'University of Caligary et le College of North Atlantic, deux universités canadiennes. De plus, le Qatar accueille des branches des universités internationales anglophones comme, par exemple Virginia Common wealth University, Georgetown University, College of London, Carnegie Mellon, Weill Cornell Medical College, Texas A\&M University, l'École des hautes études commerciales de Paris (HEC) ${ }^{8}$, etc.

L'anglais, considérée comme la lingua franca la plus prestigieuse dans le pays (Hillman 2019 : 178), est largement utilisée dans les communications quotidiennes et les transactions commerciales entre les non-arabophones et les arabophones, notamment ceux ayant un bon niveau d'études (Hillman et Ocampo Eibenschutz, 2018: 32 ; Ahmad et Hilman, 2020: 8 ; Theodoropoulou, 2020: 372). Une forme de pidgin anglais peut aussi facilement s'observer dans les échanges intercommunautaires, notamment avec les ouvriers faiblement éduqués.

\section{2. 3. Le français}

Le Qatar est un pays membre associé de l'Organisation internationale de la francophonie (OIF) depuis 2012. Le français y est considéré comme la deuxième LE, après l'anglais. Il bénéfice d'une importance croissante avec deux cent mille locuteurs francophones ${ }^{9}$, venus spécialement des pays du Maghreb.

Son enseignement a été introduit dans les écoles publiques en 2011-2012. Il est enseigné comme cours optionnel dans onze lycées publics (trois écoles pour les filles et neuf écoles pour les garçons), pendant deux ans pour les classes de Onzième et de Douzième à raison de trois heures hebdomadaires. Selon Shaikha Al-Remaihi, responsable de l'enseignement du français au ministère de l'Éducation et de l'Enseignement Supérieur qatarien, l'introduction du français dans l'enseignement secondaire du pays répond à un double objectif :

- La volonté de l'état d'offrir aux apprenants qatariens l'opportunité de connaitre une LE autre que l'anglais, afin de les préparer aux futurs métiers et aux besoins de l'État.

- Envoyer des étudiants qatariens en France afin de poursuivre leurs études dans les différents établissements de l'enseignement supérieur français ${ }^{10}$.

Quant aux écoles privées, l'enseignement du français se déroule dans des contextes variés auprès d'une population cosmopolite et généralement bilingue :

\footnotetext{
${ }^{8}$ L'enseignement dans cette école française est en anglais.

${ }^{9} \mathrm{http}$ //fipf.org/actualite/au-qatar-une-nouvelle-association-nait-loccasion-de-la-jipf

${ }^{10} \mathrm{Ces}$ informations nous ont été fournies par courrier électronique.
} 
- Français en immersion, dans le cas du lycée franco-qatarien «Voltaire ». Ce lycée est partenaire de l'AEFE (Agence pour 1'Enseignement Français à l'Etranger). Elle a été créée en 2007 et accueille des étudiants de quarante-cinq nationalités ${ }^{11}$ dont cinq cents Qatariens. Il existe également une école française « Lycée Bonaparte » ouverte depuis 1976.

- Enseignement bilingue voire trilingue (français, anglais et arabe) dans les écoles qui desservent les populations maghrébines et libanaises.

Le français est également enseigné comme cours facultatif ou obligatoire, dans une trentaine d'écoles communautaires et privées.

Pour ce qui est de l'enseignement du français au niveau universitaire, un programme de mineure de français a été créé en 2016 à l'Université du Qatar que nous présenterons dans le contexte de cette recherche. Le français est également proposé comme cours facultatif pour certaines spécialités à l'Université du Qatar, comme c'est le cas aussi au The Community College of Qatar.

Des cours ou des formations de français, à la carte, sont aussi proposés au centre de langues au Translation and Interpreting institute à Hamad Bin Khalifa University, université publique dédiée à la recherche scientifique et aux études supérieures.

Un département de français ou d'études françaises a récemment vu le jour à l'Université privée de Lusail, seule université privée qatarienne, créée en 2020-2021.

Le pays héberge également l'unique radio francophone du Golfe «Oryx Fm », une radio qatarienne créée en 2011 en partenariat avec la France.

Quant à l'emploi du français dans les communications quotidiennes, il est utilisé dans le pays entre les différentes communautés francophones. De plus, le français s'emploie aussi dans les échanges entre les résidents originaires des pays maghrébins et ceux d'autres pays arabes francophones, notamment lorsque l'écart entre les différents dialectes arabes ne permet pas une bonne communication (Benammour, 2014: 8).

\section{2. 4. Les autres $L E$}

Plusieurs autres LE pratiquées par un grand nombre de locuteurs sont aussi présentes dans le pays comme le malayalam, l'hindi, le bengali, le népali, le tamoul, le baloutchi, l'urdu, le tagalog, l'indonésien, le persan, le cinghalais, l'amharique, le pachtou et le swahili (Hillman et Ocampo Eibenschutz, 2018: 33). Ces LE appartenant à une classe sociale défavorisée n'ont aucun statut officiel dans le pays, et demeurent souvent dévalorisées. Certaines de ces langues sont enseignées dans des écoles privées communautaires telles que l'école indienne, pakistanaise, srilankaise, philippine, bengali, iranienne et éthiopienne.

Il est à noter qu'une forme parlée de l'ourdou-hindi est largement utilisée par les ouvriers asiatiques parlant le malayalam, le tamoul, le tagalog et le bengali (Ahmad et Hillman, 2020 ; Theodoropoulou, 2020).

\section{Contexte de la recherche}

Cette étude a été effectuée en 2020 auprès des étudiantes inscrites au programme de mineure de français, au sein du département de littérature anglaise et de linguistique de l'Université du Qatar. Ce programme de mineure, ouvert en 2016, offre la possibilité aux apprenantes (filles) de choisir le français comme option mineure. L'admission à ce programme est ouverte à tous les niveaux.

${ }^{11} \mathrm{https}: / /$ www.lyceevoltaire.org/presentation-du-lycee-0

XLinguae, Volume 14 Issue 4, October 2021, ISSN 1337-8384, eISSN 2453-711X 
Pour avoir leur diplôme en langue française, les apprenantes doivent obligatoirement suivre vingt-quatre heures créditées de français pendant au moins quatre semestres (deux ans). Les cours sont répartis ainsi :

- $\quad$ Six cours (18 heures) obligatoires (français I, français II, français III, expression orale I, expression écrite I, langue, culture et civilisation). Les apprenantes sont également amenées à s'inscrire en cours de laboratoire (prononciation), parallèlement avec les cours de français I et de français II, à raison de deux heures hebdomadaires non-créditées.

- Deux cours (6 heures) facultatifs, que les étudiantes peuvent choisir parmi les cours suivants : (expression orale II, expression écrite II, français des affaires, introduction à la littérature française et français pour les médias).

Les deux premiers semestres, elles suivent les cours de langue, d'expression orale et écrite niveau I. Les troisième et quatrième semestres, elles sont censées s'inscrire aux autres cours. Chaque cours est étalé sur quinze semaines pendant le semestre à raison de trois heures par semaine.

Ces apprenantes viennent de plusieurs départements et facultés au sein desquelles elles suivent leurs spécialités majeures, par exemple la littérature anglaise et la linguistique, la mass-communication, la psychologie, la chimie, les relations internationales, le droit, etc.

Le département de littérature anglaise et de linguistique offre aussi une autre mineure en espagnol, suivant plus ou moins le même schéma que le français. D'autres LE sont proposées en tant que spécialité mineure comme le turc au département d'arabe, ou en tant que cours facultatif comme le persan, le japonais ou le coréen, qui sont proposés comme matières optionnelles pour certaines spécialités à l'Université.

Il est à noter que certaines spécialités, proposées à l'Université du Qatar, sont réservées aux garçons, alors que d'autres le sont pour les filles, comme c'est le cas des programmes de langues (mineure et majeure), par exemple. En effet, les classes mixtes étant interdites à l'Université du Qatar, les spécialités proposées aux garçons et aux filles sont donc administrées dans des classes séparées.

\section{Méthodologie de la recherche}

Pour recueillir les données du terrain, nous avons distribué un questionnaire sur les représentations des étudiantes inscrites en programme de mineure de français au département de littérature anglaise et de linguistique à l'Université du Qatar. Le questionnaire se compose de deux parties :

La première partie se compose de treize questions qui visent à identifier le profil de nos sujets (âge, spécialité majeure, langue maternelle, LE connues, pratiques langagières quotidiennes, passé pédagogique) ainsi que leurs motivations.

- $\quad$ La deuxième partie, qui constitue la clé de voûte de notre recherche se compose de sept questions. Elle a pour objectif de relever les représentations que ces apprenantes se font de la langue française, de la France et des Français, ainsi que le rôle de celles-ci dans la détermination de leurs motivations et de leurs attitudes vis-à-vis de l'enseignement/apprentissage du français.

Ce questionnaire a été conçu en s'appuyant sur les critères déterminés par Dabène (1994), présentés ci-dessus. Nous avons varié entre les questions fermées et les questions ouvertes afin de permettre aux apprenantes de s'exprimer librement et d'avoir ainsi des données plus riches. 
Le questionnaire a été envoyé via BlackBoard à toutes les étudiantes inscrites dans nos différents cours proposés au premier semestre de l'année 2020 et ayant passé au moins neuf heures créditées d'apprentissage du français à l'Université. On leur a demandé de ne pas répondre plusieurs fois au même questionnaire dans les différents cours. Nous avons eu le retour de vingt-trois étudiantes sur vingt-six, et le nombre total d'étudiantes inscrites dans la mineure de français est d'environ cinquante. Les réponses de trois apprenantes étrangères (deux Tunisiennes et une Algérienne) ont été exclues de notre analyse puisque cette recherche se concentre plutôt sur les apprenantes qatariennes. Ces trois étudiantes avaient des connaissances plus approfondies en français. Elles avaient appris le français dans des écoles communautaires privées et leurs représentations ainsi que leur rapport à la langue/culture française pourraient être différentes des apprenantes qatariennes pour des raisons culturelles et sociales.

Dans le but de favoriser l'expression des apprenantes, les questions ont été rédigées en anglais et nous leur avons donné la possibilité d'y répondre en anglais, en arabe ou en français. Toutes les réponses formulées par les étudiantes ont été rédigées en anglais et nous les avons traduites en français. Le dépouillement du questionnaire s'est fait manuellement et notre méthode d'analyse était de type qualitatif. Elle se centrait alors sur le contenu en essayant de relever les éléments-clés dans la construction des représentations. Pour garder l'anonymat des apprenantes, nous leur avons attribué un code comme E1 = étudiante 1, E2 = étudiante 2.

\section{Résultats :}

Suite à l'analyse des données, nous sommes parvenus aux résultats suivants:

\section{1. Profil des répondantes}

Notre échantillon se compose de vingt étudiantes qui apprennent le français en tant que spécialité mineure. Elles sont âgées de vingt à vingt-cinq ans. Leurs niveaux en français s'échelonnent du A2 au B2 selon les niveaux de CECRL. Elles ont toutes l'arabe comme langue maternelle et maîtrisent l'anglais comme seconde langue, qu'elles utilisent simultanément avec l'arabe dans leurs communications quotidiennes. Douze étudiantes ont étudié le français pendant un à trois ans dans des écoles primaires privées ${ }^{12}$ ou dans des écoles secondaires publiques dans lesquelles le français est proposé comme cours facultatif. C'était des fausses-débutantes, et elles avaient des connaissances basiques en français avant de s'inscrire en mineure ; elles n'ont obtenu aucun diplôme en français auparavant. Les huit autres étudiantes ont commencé leur apprentissage du français à l'Université.

Douze apprenantes sont inscrites au département de littérature anglaise et de linguistique. Trois étudiantes viennent du département des Affaires internationales, trois autres du département de mass-communication et deux de la faculté de droit.

Dix enquêtées ont déjà fait des séjours en France allant de deux semaines à deux mois. En effet, la population qatarienne est l'une des plus riches du monde et beaucoup de Qatariens passent souvent leurs vacances en famille dans les pays occidentaux.

Concernant l'emploi du français en dehors de la salle de classe, la majorité des enquêtées n'ont pas souvent l'occasion de pratiquer le français en dehors de la salle de classe. Cinq étudiantes l'utilisent parfois dans leurs communications quotidiennes, comme par exemple lors des voyages dans des pays francophones, à l'institut français du Qatar, pour parler avec leurs amies francophones ou avec les membres de leurs familles, comme le note E1: "J'utilise le français avec mes

${ }^{12}$ Une de ces douze étudiantes a étudié le français au lycée franco-qatarien voltaire.

XLinguae, Volume 14 Issue 4, October 2021, ISSN 1337-8384, eISSN 2453-711X 
cousins. La plupart de mes cousins parlent français. Ils l'ont appris à l'école Voltaire. Parfois, on parle français pour le plaisir. Je parle français avec mon enseignante de français à l'école, Madame Julie. Elle est russe mais sa deuxième langue est le français et elle est actuellement une de mes meilleures amies. Je parle aussi français avec mon amie libanaise Jane ».

\section{2. Motivations}

En réponse à nos deux questions suivantes : "Pourquoi avez-vous choisi d'apprendre le français? Quelles sont vos motivations? ", nous constatons que nos enquêtées sont des apprenantes non-captives qui ont choisi volontairement d'apprendre le français pour plusieurs raisons : connaitre une nouvelle LE riche du point de vue culturel et historique, pour des raisons de prestige social, pour voyager en France, pour poursuivre des études supérieures en France ou dans des pays francophones, travailler dans des institutions publiques qatariennes où le français serait utilisé, comme au Ministère des affaires étrangères.

Ces motivations envers l'apprentissage du français peuvent être classées en deux types : des motivations internes et des motivations externes (Cuq, 2003: 171). La motivation interne (à court terme) liée au plaisir d'apprentissage, à la curiosité, à l'admiration des individus envers la langue cible et au désir de connaître sa culture et son peuple. La motivation externe, très fréquente, est considérée comme déclencheur de l'apprentissage. Elle résulte des interactions entre l'individu et les éléments extérieurs de son environnement. Dans ce type de motivation, l'apprentissage de la LE se considère comme un investissement économiquement rentable à long terme (Porcher, 1978: 33 ; Cuq, 2003: 171).

Dans notre cas, il s'agit par conséquent d'un public ayant des motivations internes et externes, ce qui doit tout naturellement développer une attitude favorable à l'apprentissage du français.

\section{3. Représentations portant sur la langue française}

Pour connaitre les représentations des apprenantes portant sur la langue française, nous leur avons posé les cinq questions suivantes: «Q1. Vos parents vous ont-ils encouragée à apprendre le français?», "Q2. Pensez-vous que l'apprentissage du français représente un avantage pour vous? Justifiez votre réponse! ", "Q3. Que représente le français pour vous? "Q4. Le français est-il une langue facile ou difficile?», "Q5. Quelles sont vos principales difficultés en français?».

Le Qatar est une société très conservatrice et les familles y sont majoritairement traditionnelles et patriarcales. L'autorité parentale, pour des raisons traditionnelles et religieuses, joue un rôle décisif dans les choix de leurs enfants y compris celui de leurs études. Il nous a donc semblé impératif de connaître l'opinion des parents de nos apprenantes sur l'apprentissage du français. En réponse à la première question, toutes les enquêtées confirment qu'elles ont eu le soutien de leurs parents pour apprendre le français. Comme l'affirment les deux enquêtées suivantes :

- E2 : "Oui, mon père m'a encouragée à apprendre le français ; c'est un homme d'affaires et il a souvent des réunions avec des Français. Je veux travailler avec lui plus tard».

- $\quad$ E3: « ma famille m'a encouragé à apprendre le français dès le début ; actuellement ils m'appellent quand ils ont besoin de comprendre quelque chose rédigé en français ».

Le Qatar entretien de très bonnes relations avec la France sur tous les plans (économique, politique, commercial, sportif, militaire, etc.). Les investissements bilatéraux entre les deux pays se sont considérablement développés ces dernières 
décennies. Conséquemment, beaucoup d'entreprises et de marques de luxe françaises se sont installées dans le pays (Carrefour, Monoprix, Sephora, Paris Gallérie, Louis Vuitton, Boucheron, Dior, Paul, La Brioche Dorée, La Fayette, etc.). L'anglais reste la première langue du commerce et des affaires dans le pays. Mais le français est de plus en plus présent et sa présence peut bien s'y développer, notamment grâce à la politique d'ouverture menée par le Qatar. Le pays cherche à diversifier son économie et le plan Qatar National Vision 2030 vise à faire du Qatar une société avancée, capable de maintenir son développement et de fournir un niveau de vie élevé à son peuple à l'horizon $2030^{13}$. Il est à noter que le fonds d'investissement souverain qatarien (Qatar Investment Authority) investit massivement en France dans plusieurs domaines tels que le sport, l'immobilier, l'hôtellerie, le luxe, etc. Cette politique de modernisation du pays et de diversification de ses ressources financières pourrait bien offrir des perspectives professionnelles aux Qatariens qui maîtrisent le français.

Les parents de nos enquêtées sont donc conscients de l'importance du français dans le pays pour la promotion professionnelle et sociale de leurs enfants. Concernant la deuxième question, la majorité de nos sujets pensent que l'apprentissage du français est considéré comme un avantage et un atout professionnels :

- E4 : «Oui, c'est un avantage pour moi, en effet toutes les langues étrangères que j'apprends sont un avantage pour moi car, après l'université, je souhaite servir mon pays et travailler au ministère des affaires étrangères pour représenter mon pays et pour être capable de communiquer avec les autres. C'est bien de parler différentes langues autres que l'anglais ».

- $\quad$ E5 : «Oui, c'est sûr parce que c'est vraiment bien de connaître deux langues et actuellement il y a plusieurs jobs qui cherchent à recruter des Qatariens qui parlent français. Cela signifie que le français est important ».

Pour certaines, il leur offre la possibilité de poursuivre leurs études supérieures dans les universités françaises, comme le note E6 : "Oui, parce que je souhaite finir mon master de droit en France » et E7 : "Absolument, la connaissance du français augmente mes chances si je veux joindre les meilleures universités en France ».

Pour réaliser le plan Qatar National Vision 2030, le pays a entamé, depuis quelques années, une politique prometteuse de qatarisation des emplois dans le pays. Dans cette optique, le gouvernement qatarien a adopté une politique très généreuse d'octroi de bourses aux étudiants qatariens. Beaucoup de Qatariens poursuivent leurs études supérieures dans les meilleurs universités et établissements de recherche dans le monde entier. De plus, l'obtention des diplômes supérieurs devient une sorte de compétition et de fierté très prestigieuse pour les familles qatariennes. Les Qatariens choisissent souvent d'étudier dans des pays anglophones comme les Etats-Unis ou le Royaume-Uni. L'enseignement/apprentissage du français s'inscrit dans cette politique et pourrait bien offrir aux étudiants qatariens la possibilité de poursuivre leurs études dans les pays francophones.

Quelques étudiantes voient que le français leur permettra de découvrir de nouvelles cultures et de communiquer avec les personnes qui parlent français au Qatar ou lors des voyages dans les pays francophones. Le français est donc considéré comme moyen de communication avec les francophones.

${ }^{13} \mathrm{https}: / /$ www.gco.gov.qa/fr/propos-du-qatar/plan-qatar-national-vision-2030/

XLinguae, Volume 14 Issue 4, October 2021, ISSN 1337-8384, eISSN 2453-711X 
Pour la troisième question, nous remarquons que le français reçoit des représentations positives de la part des enquêtées. Certaines représentations sont liées $\mathrm{au}$ statut $\mathrm{du}$ français et à son importance comme «langue importante, professionnelle », langue aux aspects culturels et historiques : "Langue de prestige, riche en culture et en littérature ». D'autres représentations sont relatives à des aspects esthétiques et affectifs «élégante, musicale, belle à entendre » :

- $\quad$ E1 : «Pour être honnête, le français est une langue élégante, musicale belle à entendre. C'est aussi une langue importante, très riche en culture et en littérature et une langue professionnelle bien sûr ».

- $\quad \mathrm{E} 8$ : "Le français est une langue de prestige. C'est aussi une langue qui attire les gens quand ils l'entendent. Tout le monde aime le français ».

Pour ce qui est des représentations linguistiques liées à l'apprentissage du français, la majorité des enquêtées (quinze étudiantes) pensent que c'est une langue difficile voire très difficile. Leurs principales difficultés en français sont la prononciation des voyelles et l'expression orale.

Bien que la présence francophone soit visible dans le pays, le français reste en situation exolingue où les apprenantes n'ont que peu d'occasions de le pratiquer en dehors de la salle de classe. En effet, pour des raisons religieuses et traditionnelles, les filles ne sortent que rarement de leurs domiciles et souvent accompagnées d'un membre de leur famille. De plus, les relations entre les Qatariens et les différentes communautés ne sont pas fréquentes, notamment pour les filles, ce qui réduit les chances de pratiquer le français en situation réelle de communication avec des natifs francophones.

Concernant la prononciation, elle constitue «la composante la plus caractéristique, la plus intime, la moins accessible d'une langue » (Defays, 2003: 40). Lors de l'apprentissage d'une LE, les rapports qui existent entre la langue maternelle des apprenants et la LC jouent un rôle fondamental dans les choix pédagogiques mis en place en classe de langue (Courtillon, 2003: 28). Le français est une langue lointaine pour un locuteur qatarien, et il existe une distance phonétique entre le français et l'arabe. L'arabe possède six voyelles alors que le français en possède seize. L'oreille des apprenantes qatariennes, qui a été habituée à discriminer six voyelles, ne perçoit pas d'emblée les onze voyelles du français qui n'existent pas en arabe. La majorité des apprenantes ne peut percevoir toutes les voyelles du français que si on met en place un mode d'entraînement à leur perception.

Conscients de ces difficultés, nous proposons deux cours de prononciation, obligatoires, non-crédités, aux apprenantes inscrites dans les deux premiers cours de français. Nous essayerons en plus chaque semestre d'organiser des activités qui leur permettent de pratiquer et d'améliorer leur niveau en français, notamment avec l'IF du Qatar.

\section{4. Représentations portant sur la France et sur les Français}

Pour identifier les représentations de nos sujets concernant la France et les Français, les enquêtées ont répondu aux deux questions suivantes : «Q6. Écrivez les cinq mots qui vous viennent à l'esprit quand vous pensez à la France! », «Q7. Écrivez les cinq mots qui vous viennent à l'esprit quand vous pensez aux Français! »

Concernant les représentations de la France, nous pouvons regrouper les réponses des apprenantes en plusieurs catégories liées :

Aux grands monuments touristiques : les champs Élysées, les musées, le Louvre, la Tour Eiffel, l'Arc de triomphe. 
- Aux personnages littéraires et historiques: Napoléon, Victor Hugo, Jean-Jacques Rousseau, etc.

- Au luxe, à la mode et aux grandes marques : Coco Channel, Louis Vuitton, La Fayette, Dior, Yves-Saint Laurent, etc.

- A la cuisine française: la pâtisserie, le croissant, la baguette, le chocolat et le fromage.

D'autres représentations sont aussi liées à la France, il en est ainsi de l'étiquette d'un beau pays à visiter et où faire du shopping.

Quant aux représentations portant sur les Français, la plupart des enquêtées en ont des positives. Pour nos sujets, les Français sont : beaux, élégants, gourmands, sérieux, simples, travailleurs, fiers de leur nationalité et de leur langue, ponctuels, bien organisés, à la mode, directs, ils aiment les voyages et la littérature.

Certaines apprenantes (deux enquêtées) ont des représentations négatives et pensent que les Français sont réservés, grossiers, lunatiques, froids, arrogants, prétentieux, toujours pressés, même hostiles et racistes.

Nous remarquons que la plupart des représentations que les enquêtées se font de la France et des Français restent classiques et fortement stéréotypées. Elles sont souvent partagées par la plupart des individus dans le monde. Ces représentations proviennent souvent des médias et pourraient être renforcées par les voyages en France.

\section{Conclusion}

Comme le souligne Boutet (1997: 28) :

Si les langues se valent toutes du point de vue du linguiste, on sait qu'il n'en va pas de même dans les représentations que les sociétés fabriquent. Des valeurs, positives ou négatives, des jugements sont associés dans chaque société aux langues qui y sont parlées.

Nous avons essayé dans ce travail d'insister sur l'importance de l'analyse des représentations des apprenants ainsi que sur l'importance de leurs motivations dans la détermination de leurs attitudes envers l'apprentissage des LE (le français dans notre cas). Les résultats de notre enquête nous ont montré que les représentations dominantes chez les apprenantes qatariennes envers la langue/culture française, la France et les Français, demeurent généralement positives. Et en conséquence, ces représentations constituent des facteurs développant des attitudes favorables à l'apprentissage du français.

Nous avons pu également constater que les apprenantes ainsi que leurs parents sont conscients de l'importance de l'apprentissage du français, de sa rentabilité et de son utilité pour leur avenir. De nos jours, on n'apprend pas une langue seulement pour le plaisir d'apprendre mais également pour des raisons économiques et professionnelles. L'apprentissage $\mathrm{du}$ français représente donc un atout professionnel important pour ces apprenantes.

Les difficultés rencontrées par les apprenantes pendant l'apprentissage du français pourraient être dépassées par la mise en place d'activités extrascolaires qui leur offrent la possibilité de perfectionner leur français en partenariat avec les différents organismes français ou francophones présents dans le pays comme l'IF du Qatar, Oryx Fm, etc.

Nous estimons que le développement de la langue française dans le pays dépend essentiellement de son statut dans les écoles publiques et nous préconisons donc d'introduire son enseignement dans les écoles en tant que cours obligatoire

XLinguae, Volume 14 Issue 4, October 2021, ISSN 1337-8384, eISSN 2453-711X 
comme deuxième LE. Nous prônons également d'élargir son enseignement à l'Université du Qatar en donnant la possibilité aux garçons de pouvoir se spécialiser en français en tant que spécialité mineure ou majeure.

Nous ne prétendons pas généraliser ces résultats au niveau du pays mais ils nous fournissent des indices importants pour comprendre la situation actuelle du français dans le pays, ainsi que les représentations qui y sont associées.

Dans nos futurs travaux de recherche, nous comptons entamer une autre étude plus approfondie et de plus grande envergure, avec pour objectif analyser les représentations des apprenants qatariens en français dans les écoles publiques du pays.

\section{Bibliographic references}

ABDALLAH-PRETCEILLE, M. 1986. Vers une pédagogie interculturelle. Paris: Institut National de Recherche pédagogique. ISBN: 0-02-926679-3.

AHMAD, R. - HILLMAN, S. 2020. Laboring to communicate: Use of migrant languages in COVID-19 awareness campaign in Qatar. In: Multilingua, Available online: https://www.degruyter.com/document/doi/10.1515/multi-2020-0119/html. Site consulté le 20 mars 2021. ISSN: 1613-3684

AIT-CHAALAL, A. 2007. Langue(s) arabe(s), monde(s) arabe(s), arabisme: éléments de réflexion et d'évaluation de dynamiques complexes. In: Revue Internationale de Politique Comparée, vol. 14, n. 1, pp. 50-68. ISSN 1370-0731

ALRABADI, E. 2012. Le FLE à l'Université du Yarmouk en Jordanie : état actuel, défis, représentations et perspectives. In: Dialogos, vol. 13, n. 26, pp. 41-52. ISSN 2734-5424

ALRABADI, E. - IRUSHEIDAT, M. 2017. Une démarche interculturelle pour l'enseignement/apprentissage du français dans le contexte universitaire jordanien. In: Dirasat : Human and social sciences, vol. 44, n. 4, pp. 256-279. ISSN: 2663-6190

ARNOLD, J. 2006. Comment les facteurs affectifs influencent-ils l'apprentissage d'une langue étrangère ? In: ELA, vol, 4, n. 144, pp 407-425. ISSN 0071-190X

AUGER, N. 2003. Ça casse la langue d'un étranger. Les représentations de la langue et de son apprentissage : une question interculturelle. In: Travaux de didactique du français langue étrangère, n. 50, pp. 23-26. ISSN 0765-1635

AVRAM, A. 2014. Immigrant workers and language formation: Gulf pidgin Arabic. In: Lengua ymigración, vol, 6, n. 2, pp. 7-40. ISSN 1889-5425

AMOSSY, R. - HERSCHBERG-Pierrot, A. 2007. Stéréotypes et clichés. Paris: Armand Colin. ISBN 2091903922

BEACCO, J-C. 2001. Les dimensions culturelles des enseignements de langue. Paris:

Hachette. ISBN 2-01-155157-9

BENAMMOUR, S. 2014. Le français à Bahreïn. In: Les Cahiers de l'Orient, vol, 2, n. 114, pp. 125-138. ISSN 0767-6468

BERGER, C. 2001. Des lycéens et l'anglais. Rôle des variables socioculturelles dans la représentation des pays anglophones. Gloria Paganini (coord.). Différences et proximités culturelles : l'Europe. Paris: L'Harmattan, pp. 39-52. ISBN : 2-7475-01973

BILLIEZ, J. - MILLET, A. 2001. Représentations sociales: Trajets théoriques et méthodologiques. Daniele Moore (coord.). Pairs : Didier, pp. 31-50. ISBN 978-2-27805032-1.

BOUTET, J. 1997. Langage et société. Paris: Seuil. ISBN 2020298732

CONSEIL DE L'EUROPE. 2002. Cadre européen commun de référence pour les langues : apprendre, enseigner, évaluer. Strasbourg/Paris: Didier. ISBN 2278050753

CAIN, A. - DE PIETRO, J-F. 1997. Les représentations des pays dont on apprend la langue : complément facultatif ou composante de l'apprentissage ? Marinette Matthey (éd.). Les langues et leurs images: actes du colloque, Neuchâtel: IRDP, pp. 300-307. ISBN 978-2-606-00498-9 
CALVET, L-J. 1999. Pour une écologie des langues du monde. Paris: Plon. ISBN 225918975X

CALVET, L-J. 2007. La mondialisation au filtre des traductions. In: Hermès, vol. 3, n. 49, pp. 45-57. ISSN 0767-9513

CANDELIER, M. - HERMANN-BRENNEKE, G. 1993. Entre le choix de l'abandon : les langues étrangères à l'école, vues d'Allemagne et de France. Paris : Didier. ISBN 978-2-278-04364-4

CANDELIER, M. 1997. Catégoriser les représentations. Genvieve Zarate et Michel Candelier (dirs.) Les représentations en didactique des langues et cultures. Paris: ENS, pp, 43-65. ISBN 2905769335

CASTELlOTTI, V. - MOORE, D. 2002. Représentation sociales des langues et enseignements. Strasbourg : conseil de l'Europe.

CAVALLI, M. - COLETTA, D. 2002. Langues, bilinguisme et représentations sociales au Val d'Aoste, Aoste : IRRE. ISSN 1131547593

COURTILLON, J. 2003. Comment élaborer un cours de FLE. Paris: Hachette. ISSN 978-2-01-155214-3.

DABENE, L. 1994. Repères sociolinguistiques pour l'enseignement des langues. Paris: Hachette. ISBN 2010206479

DABENE, L. 1997. L'image des langues et de leur apprentissage. Matthey M. (éd.). Les langues et leurs images. Neuchâtel: IRDP, pp 19-23. ISSN 978-2-606-00498-9

DEFAYS, J-M. 2003. Le français langue étrangère et seconde : enseignement et apprentissage. Liège: Mardaga. ISBN 2870098464

GOHARD-RADENKOVI - DENISE LUSSIER, A. - PENZ, H. - ZARATE, G. 2003. La médiation culturelle en didactique des langues comme processus. Strasbourg: Conseil de L'Europe. ISBN ISBN 92-871-5259-4

HILLMAN, S. - OCAMPO EIBENSCHUTZ, E. 2018. English, super-diversity, and identity in the State of Qatar. In: World Englishes n. 37, vol, 2, pp. 228-247. ISSN 1467-971X

HILLMAN, S. 2019. It will be funny [to speak Hindi]: Travelling Englishes and perceptions about learning migrant languages in Qatar. In: Transitions: Journal of Transient Migration Intellect, vol, 3, n. 3, pp, 177-200. ISSN 23977140

JODELET, D. 1989. Représentations sociales : un domaine en expansion. Paris: PUF. ISBN 978-2-13-053765-6

MATTHEY, M. 1997. Les langues et leurs images, Neuchâtel: IRDP. ISSN 978-2606-00498-9

MATTHEY, M. 2000. Les représentations de l'apprentissage des langues et du bilinguisme dans l'institution éducative." In: ELA, n. 120, pp. 487-496. ISSN 0071190x.

MOORE, D. 2001. Les représentations des langues et de leur apprentissage : itinéraires théoriques et trajets méthodologiques." Danièle Moore (coord.). Les représentations des langues et de leur apprentissage : Référence, modèles, données et méthodes. Paris: Didier, pp. 7-22. ISBN 978-2-278-05032-1

MOSCOVICI, S. 1989. Des représentations collectives aux représentations sociales: éléments pour une histoire. Danièle Jodelet (éd.). Les représentations sociales. Paris: PUF. ISBN 978-2-13-053765-6

MULLER, N. 1998. L'allemand, c'est pas du français ! : Enjeux et paradoxes de l'apprentissage de l'allemand. Neuchâtel: INRP-LEP. ISBN 2-606-00643-7

MULLER, N - DE PIETRO, J-F. 2001. Que faire de la notion de représentations ? Danièle Moore (éd.). Les représentations des langues et leur apprentissage : références, modèles, données et méthodes. Paris: Didier, pp. 51-64. ISBN 978-2-27805032-1

NEBEL, A. 2017. Linguistic Superdiversity and English-Medium Higher Education in Qatar. 27-40. L. Arnold, A. Nebel, \& L. Ronesi (eds.). Emerging writing research

XLinguae, Volume 14 Issue 4, October 2021, ISSN 1337-8384, eISSN 2453-711X 
from the Middle East-North Africa region.. Fort Collins, CO: The WAC Clearinghouse and University Press of Colorado, pp. 27-40. ISBN 978-1-64215-0896

PAGANINI, G. 1998. Entre le "très proche » et le «pas assez loin » : différences, proximité et représentation de l'italien en France. Thèse de doctorat, Paris III.

PORCHER, L. 1987. Champs de signes, Etats de la diffusion du français langue étrangère. Paris: Crédif/Didier. ISBN 2-278-03749-8

PORCHER, L. 1995. Le français langue étrangère. Paris: Hachette. ISBN 201170409X.

PUREN, C. 1997. Civilisation et didactique des langues: les différentes orientations de l'approche interculturelle. Available online: https://www.christianpuren.com/mestravaux/.

PY, B. 2004. Pour une approche linguistique des approches linguistique des représentations. In: Langages, vol, 2, n. 154, pp. 6-19. ISBN 9782035770714

RICHTERICHT, R. 1985. Besoins langagiers et objectifs d'apprentissage. Paris: Hachette. ISBN 2010078071

SROUR-GANDON, P. 2013. La stratégie économique du Qatar. In: Confluences Méditerranée, vol. 1, n. 84, pp. 45-57. ISBN 9782343003054

THEODOROPOULOU, I. 2020. Blue-collar workplace communicative practices: A case study in construction sites in Qatar. In: Language Policy, n. 19, pp 363-387. ISSN 1568-4555

ZARATE, G. 1986. Enseigner une culture étrangère. Paris: Hachette. ISBN 2.01.011872.3

ZARATE, G. 1993. Les représentations de l'étranger et didactique des langues. Paris: Crédif/Hatier. ISBN 978-2-278-04311-8

ZARATE, G. 1997. La notion de représentation et ses déclinaisons. In: Notions en question, n. 2, pp. 5-9. ISBN 2-905769-33-5

Available online:

https://www.psa.gov.qa/en/statistics1/pages/default.aspx

https://priyadsouza.com/population-of-qatar-by-nationality-in-2017/

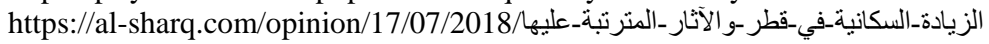

https://www.tresor.economie.gouv.fr/Articles/a7d5a8e0-fc0b-4ae2-acda-

3efa5c8e9fce/files/be882ed0-a42a-49fe-b269-569b95dd629f

https://www.monde-diplomatique.fr/2018/10/MOUNIER_KUHN/59150

http://fipf.org/actualite/au-qatar-une-nouvelle-association-nait-loccasion-de-la-jipf https://www.lyceevoltaire.org/presentation-du-lycee-0

https://diwan.gov.qa/briefing-room/news/general/lg/2019/140119/lg02?sc_lang=en

https://www.gco.gov.qa/fr/propos-du-qatar/plan-qatar-national-vision-2030/

Words: 7891

Characters: 54187 (30,10 standard pages)

Assoc. prof. Elie Alrabadi, PhD

French Minor Program

Department of English Literature and linguistics

College of Arts and Sciences, Qatar University

2713, Doha

Qatar

elierabad@qu.edu.qa 